\title{
Model-Independent Measurement of the $W$-Boson Helicity in Top-Quark Decays at D0
}

V. M. Abazov, ${ }^{36}$ B. Abbott, ${ }^{76}$ M. Abolins,${ }^{66}$ B. S. Acharya, ${ }^{29}$ M. Adams, ${ }^{52}$ T. Adams,${ }^{50}$ E. Aguilo, ${ }^{6}$ S. H. Ahn, ${ }^{31}$ M. Ahsan, ${ }^{60}$ G. D. Alexeev, ${ }^{36}$ G. Alkhazov, ${ }^{40}$ A. Alton, ${ }^{65}$, G. Alverson, ${ }^{64}$ G. A. Alves, ${ }^{2}$ M. Anastasoaie,${ }^{35}$ L. S. Ancu, ${ }^{35}$ T. Andeen, ${ }^{54}$ S. Anderson, ${ }^{46}$ B. Andrieu, ${ }^{17}$ M. S. Anzelc,${ }^{54}$ Y. Arnoud, ${ }^{14}$ M. Arov, ${ }^{61}$ M. Arthaud ${ }^{18}$ A. Askew, ${ }^{50}$ B. Åsman, ${ }^{41}$ A. C. S. Assis Jesus, ${ }^{3}$ O. Atramentov,${ }^{50}$ C. Autermann, ${ }^{21}$ C. Avila, ${ }^{8}$ C. Ay, ${ }^{24}$ F. Badaud, ${ }^{13}$ A. Baden, ${ }^{62}$ L. Bagby, ${ }^{53}$ B. Baldin,${ }^{51}$ D. V. Bandurin, ${ }^{60}$ S. Banerjee, ${ }^{29}$ P. Banerjee,${ }^{29}$ E. Barberis, ${ }^{64}$ A.-F. Barfuss, ${ }^{15}$ P. Bargassa, ${ }^{81}$

P. Baringer, ${ }^{59}$ J. Barreto, ${ }^{2}$ J. F. Bartlett, ${ }^{51}$ U. Bassler, ${ }^{18}$ D. Bauer,${ }^{44}$ S. Beale ${ }^{6}$ A. Bean,${ }^{59}$ M. Begalli, ${ }^{3}$ M. Begel,${ }^{72}$ C. Belanger-Champagne, ${ }^{41}$ L. Bellantoni, ${ }^{51}$ A. Bellavance, ${ }^{51}$ J. A. Benitez ${ }^{66}$ S. B. Beri, ${ }^{27}$ G. Bernardi,${ }^{17}$ R. Bernhard ${ }^{23}$ I. Bertram, ${ }^{43}$ M. Besançon, ${ }^{18}$ R. Beuselinck,${ }^{44}$ V. A. Bezzubov, ${ }^{39}$ P. C. Bhat,${ }^{51}$ V. Bhatnagar, ${ }^{27}$ C. Biscarat,${ }^{20}$ G. Blazey, ${ }^{53}$ F. Blekman, ${ }^{44}$ S. Blessing, ${ }^{50}$ D. Bloch,${ }^{19}$ K. Bloom, ${ }^{68}$ A. Boehnlein, ${ }^{51}$ D. Boline, ${ }^{63}$ T. A. Bolton, ${ }^{60}$ G. Borissov,${ }^{43}$

T. Bose, ${ }^{78}$ A. Brandt, ${ }^{79}$ R. Brock, ${ }^{66}$ G. Brooijmans, ${ }^{71}$ A. Bross, ${ }^{51}$ D. Brown, ${ }^{82}$ N. J. Buchanan, ${ }^{50}$ D. Buchholz,${ }^{54}$ M. Buehler, ${ }^{82}$ V. Buescher, ${ }^{22}$ V. Bunichev, ${ }^{38}$ S. Burdin, ${ }^{43, \dagger}$ S. Burke, ${ }^{46}$ T. H. Burnett, ${ }^{83}$ C. P. Buszello, ${ }^{44}$ J. M. Butler, ${ }^{63}$ P. Calfayan, ${ }^{25}$ S. Calvet,${ }^{16}$ J. Cammin, ${ }^{72}$ W. Carvalho, ${ }^{3}$ B. C. K. Casey,${ }^{51}$ N. M. Cason, ${ }^{56}$ H. Castilla-Valdez, ${ }^{33}$ S. Chakrabarti, ${ }^{18}$ D. Chakraborty, ${ }^{53}$ K. M. Chan, ${ }^{56}$ K. Chan, ${ }^{6}$ A. Chandra, ${ }^{49}$ F. Charles, ${ }^{19, \pi}$ E. Cheu, ${ }^{46}$ F. Chevallier, ${ }^{14}$ D. K. Cho, ${ }^{63}$ S. Choi,${ }^{32}$ B. Choudhary, ${ }^{28}$ L. Christofek, ${ }^{78}$ T. Christoudias,${ }^{44}$ S. Cihangir, ${ }^{51}$ D. Claes, ${ }^{68}$ Y. Coadou, ${ }^{6}$ M. Cooke, ${ }^{81}$ W. E. Cooper,${ }^{51}$ M. Corcoran,${ }^{81}$ F. Couderc, ${ }^{18}$ M.-C. Cousinou, ${ }^{15}$ S. Crépé-Renaudin,,${ }^{14}$ D. Cutts, ${ }^{78}$ M. Ćwiok, ${ }^{30}$ H. da Motta, ${ }^{2}$ A. Das,${ }^{46}$ G. Davies,${ }^{44}$ K. De ${ }^{79}$ S. J. de Jong, ${ }^{35}$ E. De La Cruz-Burelo, ${ }^{65}$

C. De Oliveira Martins, ${ }^{3}$ J. D. Degenhardt, ${ }^{65}$ F. Déliot, ${ }^{18}$ M. Demarteau,${ }^{51}$ R. Demina, ${ }^{72}$ D. Denisov,${ }^{51}$ S. P. Denisov, ${ }^{39}$ S. Desai, ${ }^{51}$ H. T. Diehl, ${ }^{51}$ M. Diesburg, ${ }^{51}$ A. Dominguez, ${ }^{68}$ H. Dong, ${ }^{73}$ L. V. Dudko, ${ }^{38}$ L. Duflot, ${ }^{16}$ S. R. Dugad, ${ }^{29}$ D. Duggan, ${ }^{50}$ A. Duperrin, ${ }^{15}$ J. Dyer ${ }^{66}$ A. Dyshkant,${ }^{53}$ M. Eads,${ }^{68}$ D. Edmunds,${ }^{66}$ J. Ellison, ${ }^{49}$ V. D. Elvira ${ }^{51}$ Y. Enari,${ }^{78}$ S. Eno, ${ }^{62}$ P. Ermolov, ${ }^{38}$ H. Evans, ${ }^{55}$ A. Evdokimov, ${ }^{74}$ V. N. Evdokimov,${ }^{39}$ A. V. Ferapontov,${ }^{60}$ T. Ferbel, ${ }^{72}$ F. Fiedler, ${ }^{24}$ F. Filthaut, ${ }^{35}$ W. Fisher, ${ }^{51}$ H. E. Fisk,${ }^{51}$ M. Ford,${ }^{45}$ M. Fortner, ${ }^{53}$ H. Fox ${ }^{23}$ S. Fu,${ }^{51}$ S. Fuess, ${ }^{51}$ T. Gadfort,${ }^{71}$ C. F. Galea, ${ }^{35}$ E. Gallas, ${ }^{51}$ E. Galyaev, ${ }^{56}$ C. Garcia, ${ }^{72}$ A. Garcia-Bellido, ${ }^{83}$ V. Gavrilov,${ }^{37}$ P. Gay ${ }^{13}$ W. Geist, ${ }^{19}$ D. Gelé, ${ }^{19}$ C. E. Gerber, ${ }^{52}$ Y. Gershtein, ${ }^{50}$ D. Gillberg, ${ }^{6}$ G. Ginther, ${ }^{72}$ N. Gollub ${ }^{41}$ B. Gómez, ${ }^{8}$ A. Goussiou, ${ }^{56}$ P. D. Grannis, ${ }^{73}$ H. Greenlee, ${ }^{51}$ Z. D. Greenwood, ${ }^{61}$ E. M. Gregores,${ }^{4}$ G. Grenier ${ }^{20}$ Ph. Gris, ${ }^{13}$ J.-F. Grivaz, ${ }^{16}$ A. Grohsjean ${ }^{25}$ S. Grünendahl, ${ }^{51}$ M. W. Grünewald ${ }^{30}$ J. Guo, ${ }^{73}$ F. Guo, ${ }^{73}$ P. Gutierrez,${ }^{76}$ G. Gutierrez, ${ }^{51}$ A. Haas, ${ }^{71}$ N. J. Hadley, ${ }^{62}$ P. Haefner,${ }^{25}$ S. Hagopian, ${ }^{50}$ J. Haley, ${ }^{69}$ I. Hall, ${ }^{66}$ R. E. Hall, ${ }^{48}$ L. Han, ${ }^{7}$ P. Hansson, ${ }^{41}$ K. Harder, ${ }^{45}$ A. Harel, ${ }^{72}$ R. Harrington, ${ }^{64}$ J. M. Hauptman, ${ }^{58}$ R. Hauser, ${ }^{66}$ J. Hays,${ }^{44}$ T. Hebbeker, ${ }^{21}$ D. Hedin, ${ }^{53}$ J. G. Hegeman, ${ }^{34}$ J. M. Heinmiller, ${ }^{52}$ A. P. Heinson, ${ }^{49}$ U. Heintz, ${ }^{63}$ C. Hensel,,${ }^{59}$ K. Herner, ${ }^{73}$ G. Hesketh, ${ }^{64}$ M. D. Hildreth, ${ }^{56}$ R. Hirosky, ${ }^{82}$ J. D. Hobbs, ${ }^{73}$ B. Hoeneisen, ${ }^{12}$

H. Hoeth, ${ }^{26}$ M. Hohlfeld, ${ }^{22}$ S. J. Hong, ${ }^{31}$ S. Hossain, ${ }^{76}$ P. Houben,${ }^{34}$ Y. Hu, ${ }^{73}$ Z. Hubacek, ${ }^{10}$ V. Hynek, ${ }^{9}$ I. Iashvili, ${ }^{70}$

R. Illingworth, ${ }^{51}$ A. S. Ito, ${ }^{51}$ S. Jabeen, ${ }^{63}$ M. Jaffré, ${ }^{16}$ S. Jain, ${ }^{76}$ K. Jakobs,${ }^{23}$ C. Jarvis, ${ }^{62}$ R. Jesik, ${ }^{44}$ K. Johns, ${ }^{46}$

C. Johnson, ${ }^{71}$ M. Johnson, ${ }^{51}$ A. Jonckheere, ${ }^{51}$ P. Jonsson, ${ }^{44}$ A. Juste, ${ }^{51}$ E. Kajfasz,${ }^{15}$ A. M. Kalinin, ${ }^{36}$ J. R. Kalk, ${ }^{66}$

J. M. Kalk, ${ }^{61}$ S. Kappler, ${ }^{21}$ D. Karmanov,${ }^{38}$ P. A. Kasper,${ }^{51}$ I. Katsanos,${ }^{71}$ D. Kau, ${ }^{50}$ R. Kaur, ${ }^{27}$ V. Kaushik, ${ }^{79}$ R. Kehoe, ${ }^{80}$

S. Kermiche, ${ }^{15}$ N. Khalatyan, ${ }^{51}$ A. Khanov, ${ }^{77}$ A. Kharchilava, ${ }^{70}$ Y. M. Kharzheev, ${ }^{36}$ D. Khatidze, ${ }^{71}$ T. J. Kim, ${ }^{31}$ M. H. Kirby ${ }^{54}$ M. Kirsch ${ }^{21}$ B. Klima, ${ }^{51}$ J. M. Kohli, ${ }^{27}$ J.-P. Konrath ${ }^{23}$ V. M. Korablev, ${ }^{39}$ A. V. Kozelov, ${ }^{39}$ D. Krop,${ }^{55}$

T. Kuhl, ${ }^{24}$ A. Kumar, ${ }^{70}$ S. Kunori, ${ }^{62}$ A. Kupco, ${ }^{11}$ T. Kurča, ${ }^{20}$ J. Kvita, ${ }^{9}$ F. Lacroix,${ }^{13}$ D. Lam,${ }^{56}$ S. Lammers, ${ }^{71}$ G. Landsberg, ${ }^{78}$ P. Lebrun, ${ }^{20}$ W. M. Lee, ${ }^{51}$ A. Leflat, ${ }^{38}$ F. Lehner, ${ }^{42}$ J. Lellouch,${ }^{17}$ J. Leveque, ${ }^{46}$ J. Li, ${ }^{79}$ Q. Z. Li,${ }^{51}$ L. Li, ${ }^{49}$ S. M. Lietti, ${ }^{5}$ J. G. R. Lima, ${ }^{53}$ D. Lincoln,${ }^{51}$ J. Linnemann, ${ }^{66}$ V. V. Lipaev, ${ }^{39}$ R. Lipton, ${ }^{51}$ Y. Liu, ${ }^{7}$ Z. Liu,,${ }^{6}$ A. Lobodenko, ${ }^{40}$ M. Lokajicek, ${ }^{11}$ P. Love, ${ }^{43}$ H. J. Lubatti,${ }^{83}$ R. Luna, ${ }^{3}$ A. L. Lyon, ${ }^{51}$ A. K. A. Maciel, ${ }^{2}$ D. Mackin, ${ }^{81}$ R. J. Madaras, ${ }^{47}$ P. Mättig, ${ }^{26}$ C. Magass, ${ }^{21}$ A. Magerkurth, ${ }^{65}$ P. K. Mal, ${ }^{56}$ H. B. Malbouisson, ${ }^{3}$ S. Malik, ${ }^{68}$ V. L. Malyshev, ${ }^{36}$ H. S. Mao, ${ }^{51}$ Y. Maravin, ${ }^{60}$ B. Martin, ${ }^{14}$ R. McCarthy, ${ }^{73}$ A. Melnitchouk, ${ }^{67}$ L. Mendoza, ${ }^{8}$ P. G. Mercadante, ${ }^{5}$ M. Merkin, ${ }^{38}$

K. W. Merritt, ${ }^{51}$ J. Meyer, ${ }^{22,8}$ A. Meyer, ${ }^{21}$ T. Millet, ${ }^{20}$ J. Mitrevski, ${ }^{71}$ J. Molina, ${ }^{3}$ R. K. Mommsen, ${ }^{45}$ N. K. Mondal, ${ }^{29}$

R. W. Moore, ${ }^{6}$ T. Moulik, ${ }^{59}$ G. S. Muanza, ${ }^{20}$ M. Mulders, ${ }^{51}$ M. Mulhearn, ${ }^{71}$ O. Mundal, ${ }^{22}$ L. Mundim, ${ }^{3}$ E. Nagy, ${ }^{15}$ M. Naimuddin, ${ }^{51}$ M. Narain, ${ }^{78}$ N. A. Naumann, ${ }^{35}$ H. A. Neal, ${ }^{65}$ J. P. Negret, ${ }^{8}$ P. Neustroev, ${ }^{40}$ H. Nilsen,${ }^{23}$ H. Nogima, ${ }^{3}$ S. F. Novaes, ${ }^{5}$ T. Nunnemann, ${ }^{25}$ V. O’Dell, ${ }^{51}$ D. C. O'Neil,${ }^{6}$ G. Obrant,${ }^{40}$ C. Ochando,${ }^{16}$ D. Onoprienko,${ }^{60}$ N. Oshima,${ }^{51}$

J. Osta, ${ }^{56}$ R. Otec, ${ }^{10}$ G. J. Otero y Garzón, ${ }^{51}$ M. Owen, ${ }^{45}$ P. Padley, ${ }^{81}$ M. Pangilinan, ${ }^{78}$ N. Parashar, ${ }^{57}$ S.-J. Park, ${ }^{72}$

S. K. Park,${ }^{31}$ J. Parsons,${ }^{71}$ R. Partridge,${ }^{78}$ N. Parua,${ }^{55}$ A. Patwa, ${ }^{74}$ G. Pawloski,${ }^{81}$ B. Penning, ${ }^{23}$ M. Perfilov, ${ }^{38}$ K. Peters,${ }^{45}$ Y. Peters, ${ }^{26}$ P. Pétroff,${ }^{16}$ M. Petteni, ${ }^{44}$ R. Piegaia,${ }^{1}$ J. Piper, ${ }^{66}$ M.-A. Pleier, ${ }^{22}$ P. L. M. Podesta-Lerma,${ }^{33,}$ V. M. Podstavkov, ${ }^{51}$ Y. Pogorelov, ${ }^{56}$ M.-E. Pol, ${ }^{2}$ P. Polozov,${ }^{37}$ B. G. Pope,${ }^{66}$ A. V. Popov, ${ }^{39}$ C. Potter,${ }^{6}$ 
W. L. Prado da Silva, ${ }^{3}$ H. B. Prosper, ${ }^{50}$ S. Protopopescu, ${ }^{74}$ J. Qian,${ }^{65}$ A. Quadt,${ }^{22,8}$ B. Quinn,,${ }^{67}$ A. Rakitine,${ }^{43}$ M. S. Rangel, ${ }^{2}$ K. Ranjan, ${ }^{28}$ P. N. Ratoff,${ }^{43}$ P. Renkel, ${ }^{80}$ S. Reucroft,${ }^{64}$ P. Rich,${ }^{45}$ J. Rieger,${ }^{55}$ M. Rijssenbeek ${ }^{73}$ I. Ripp-Baudot, ${ }^{19}$ F. Rizatdinova, ${ }^{77}$ S. Robinson, ${ }^{44}$ R. F. Rodrigues, ${ }^{3}$ M. Rominsky, ${ }^{76}$ C. Royon, ${ }^{18}$ P. Rubinov,${ }^{51}$ R. Ruchti, ${ }^{56}$ G. Safronov, ${ }^{37}$ G. Sajot, ${ }^{14}$ A. Sánchez-Hernández,${ }^{33}$ M. P. Sanders, ${ }^{17}$ A. Santoro, ${ }^{3}$ G. Savage,${ }^{51}$ L. Sawyer, ${ }^{61}$ T. Scanlon, ${ }^{44}$ D. Schaile, ${ }^{25}$ R. D. Schamberger,${ }^{73}$ Y. Scheglov, ${ }^{40}$ H. Schellman, ${ }^{54}$ T. Schliephake, ${ }^{26}$ C. Schwanenberger ${ }^{45}$ A. Schwartzman, ${ }^{69}$ R. Schwienhorst, ${ }^{66}$ J. Sekaric, ${ }^{50}$ H. Severini, ${ }^{76}$ E. Shabalina,${ }^{52}$ M. Shamim, ${ }^{60}$ V. Shary, ${ }^{18}$ A. A. Shchukin, ${ }^{39}$ R. K. Shivpuri, ${ }^{28}$ V. Siccardi, ${ }^{19}$ V. Simak, ${ }^{10}$ V. Sirotenko, ${ }^{51}$ P. Skubic, ${ }^{76}$ P. Slattery, ${ }^{72}$ D. Smirnov, ${ }^{56}$ J. Snow, ${ }^{75}$ G. R. Snow, ${ }^{68}$ S. Snyder, ${ }^{74}$ S. Söldner-Rembold, ${ }^{45}$ L. Sonnenschein, ${ }^{17}$ A. Sopczak, ${ }^{43}$ M. Sosebee, ${ }^{79}$ K. Soustruznik, ${ }^{9}$ B. Spurlock, ${ }^{79}$ J. Stark,,${ }^{14}$ J. Steele, ${ }^{61}$ V. Stolin, ${ }^{37}$ D. A. Stoyanova, ${ }^{39}$ J. Strandberg, ${ }^{65}$ S. Strandberg, ${ }^{41}$ M. A. Strang ${ }^{70}$ M. Strauss,${ }^{76}$ E. Strauss, ${ }^{73}$ R. Ströhmer ${ }^{25}$ D. Strom,${ }^{54}$ L. Stutte,${ }^{51}$ S. Sumowidagdo,${ }^{50}$ P. Svoisky, ${ }^{56}$ A. Sznajder, ${ }^{3}$ M. Talby, ${ }^{15}$ P. Tamburello, ${ }^{46}$ A. Tanasijczuk, ${ }^{1}$ W. Taylor, ${ }^{6}$ J. Temple,${ }^{46}$ B. Tiller, ${ }^{25}$ F. Tissandier,${ }^{13}$ M. Titov, ${ }^{18}$ V. V. Tokmenin, ${ }^{36}$ T. Toole,${ }^{62}$ I. Torchiani, ${ }^{23}$ T. Trefzger, ${ }^{24}$ D. Tsybychev, ${ }^{73}$ B. Tuchming,${ }^{18}$ C. Tully, ${ }^{69}$ P. M. Tuts, ${ }^{71}$

R. Unalan, ${ }^{66}$ S. Uvarov, ${ }^{40}$ L. Uvarov ${ }^{40}$ S. Uzunyan, ${ }^{53}$ B. Vachon,,${ }^{6}$ P. J. van den Berg, ${ }^{34}$ R. Van Kooten, ${ }^{55}$ W. M. van Leeuwen,${ }^{34}$ N. Varelas, ${ }^{52}$ E. W. Varnes, ${ }^{46}$ I. A. Vasilyev, ${ }^{39}$ M. Vaupel,${ }^{26}$ P. Verdier, ${ }^{20}$ L. S. Vertogradov,${ }^{36}$ M. Verzocchi, ${ }^{51}$ F. Villeneuve-Seguier, ${ }^{44}$ P. Vint, ${ }^{44}$ P. Vokac, ${ }^{10}$ E. Von Toerne,${ }^{60}$ M. Voutilainen, ${ }^{68, \|}$ R. Wagner,${ }^{69}$ H. D. Wahl, ${ }^{50}$ L. Wang, ${ }^{62}$ M. H. L. S Wang, ${ }^{51}$ J. Warchol,${ }^{56}$ G. Watts, ${ }^{83}$ M. Wayne, ${ }^{56}$ M. Weber,${ }^{51}$ G. Weber, ${ }^{24}$ L. Welty-Rieger, ${ }^{55}$ A. Wenger, ${ }^{42}$ N. Wermes,${ }^{22}$ M. Wetstein, ${ }^{62}$ A. White, ${ }^{79}$ D. Wicke, ${ }^{26}$ G. W. Wilson, ${ }^{59}$ S. J. Wimpenny, ${ }^{49}$ M. Wobisch, ${ }^{61}$ D. R. Wood, ${ }^{64}$ T. R. Wyatt, ${ }^{45}$ Y. Xie,${ }^{78}$ S. Yacoob,${ }^{54}$ R. Yamada,${ }^{51}$ M. Yan, ${ }^{62}$ T. Yasuda,${ }^{51}$

Y. A. Yatsunenko, ${ }^{36}$ K. Yip,${ }^{74}$ H. D. Yoo ${ }^{78}$ S. W. Youn, ${ }^{54}$ J. Yu, ${ }^{79}$ A. Zatserklyaniy,,${ }^{53}$ C. Zeitnitz ${ }^{26}$ T. Zhao, ${ }^{83}$ B. Zhou, ${ }^{65}$ J. Zhu, ${ }^{73}$ M. Zielinski, ${ }^{72}$ D. Zieminska, ${ }^{55}$ A. Zieminski, ${ }^{55,4}$ L. Zivkovic, ${ }^{71}$ V. Zutshi, ${ }^{53}$ and E. G. Zverev ${ }^{38}$

\title{
(D0 Collaboration)
}

\author{
${ }^{1}$ Universidad de Buenos Aires, Buenos Aires, Argentina \\ ${ }^{2}$ LAFEX, Centro Brasileiro de Pesquisas Físicas, Rio de Janeiro, Brazil \\ ${ }^{3}$ Universidade do Estado do Rio de Janeiro, Rio de Janeiro, Brazil \\ ${ }^{4}$ Universidade Federal do ABC, Santo André, Brazil \\ ${ }^{5}$ Instituto de Física Teórica, Universidade Estadual Paulista, São Paulo, Brazil \\ ${ }^{6}$ University of Alberta, Edmonton, Alberta, Canada, Simon Fraser University, Burnaby, British Columbia, Canada, \\ York University, Toronto, Ontario, Canada, \\ and McGill University, Montreal, Quebec, Canada \\ ${ }^{7}$ University of Science and Technology of China, Hefei, People's Republic of China \\ ${ }^{8}$ Universidad de los Andes, Bogotá, Colombia \\ ${ }^{9}$ Center for Particle Physics, Charles University, Prague, Czech Republic \\ ${ }^{10}$ Czech Technical University, Prague, Czech Republic \\ ${ }^{11}$ Center for Particle Physics, Institute of Physics, Academy of Sciences of the Czech Republic, Prague, Czech Republic \\ ${ }^{12}$ Universidad San Francisco de Quito, Quito, Ecuador \\ ${ }^{13}$ LPC, Univ Blaise Pascal, CNRS/IN2P3, Clermont, France \\ ${ }^{14}$ LPSC, Université Joseph Fourier Grenoble 1, CNRS/IN2P3, Institut National Polytechnique de Grenoble, France \\ ${ }^{15}$ CPPM, IN2P3/CNRS, Université de la Méditerranée, Marseille, France \\ ${ }^{16}$ LAL, Univ Paris-Sud, IN2P3/CNRS, Orsay, France \\ ${ }^{17}$ LPNHE, IN2P3/CNRS, Universités Paris VI and VII, Paris, France \\ ${ }^{18}$ DAPNIA/Service de Physique des Particules, CEA, Saclay, France \\ ${ }^{19}$ IPHC, Université Louis Pasteur et Université de Haute Alsace, CNRS/IN2P3, Strasbourg, France \\ ${ }^{20} I P N L$, Université Lyon 1, CNRS/IN2P3, Villeurbanne, France and Université de Lyon, Lyon, France \\ ${ }^{21}$ III. Physikalisches Institut A, RWTH Aachen, Aachen, Germany \\ ${ }^{22}$ Physikalisches Institut, Universität Bonn, Bonn, Germany \\ ${ }^{23}$ Physikalisches Institut, Universität Freiburg, Freiburg, Germany \\ ${ }^{24}$ Institut für Physik, Universität Mainz, Mainz, Germany \\ ${ }^{25}$ Ludwig-Maximilians-Universität München, München, Germany \\ ${ }^{26}$ Fachbereich Physik, University of Wuppertal, Wuppertal, Germany \\ ${ }^{27}$ Panjab University, Chandigarh, India \\ ${ }^{28}$ Delhi University, Delhi, India \\ ${ }^{29}$ Tata Institute of Fundamental Research, Mumbai, India \\ ${ }^{30}$ University College Dublin, Dublin, Ireland
}


${ }^{31}$ Korea Detector Laboratory, Korea University, Seoul, Korea

${ }^{32}$ SungKyunKwan University, Suwon, Korea

${ }^{33}$ CINVESTAV, Mexico City, Mexico

${ }^{34}$ FOM-Institute NIKHEF and University of Amsterdam/NIKHEF, Amsterdam, The Netherlands

${ }^{35}$ Radboud University Nijmegen/NIKHEF, Nijmegen, The Netherlands

${ }^{36}$ Joint Institute for Nuclear Research, Dubna, Russia

${ }^{37}$ Institute for Theoretical and Experimental Physics, Moscow, Russia

${ }^{38}$ Moscow State University, Moscow, Russia

${ }^{39}$ Institute for High Energy Physics, Protvino, Russia

${ }^{40}$ Petersburg Nuclear Physics Institute, St. Petersburg, Russia

${ }^{41}$ Lund University, Lund, Sweden, Royal Institute of Technology and Stockholm University, Stockholm, Sweden, and Uppsala University, Uppsala, Sweden

${ }^{42}$ Physik Institut der Universität Zürich, Zürich, Switzerland

${ }^{43}$ Lancaster University, Lancaster, United Kingdom

${ }^{44}$ Imperial College, London, United Kingdom

${ }^{45}$ University of Manchester, Manchester, United Kingdom

${ }^{46}$ University of Arizona, Tucson, Arizona 85721, USA

${ }^{47}$ Lawrence Berkeley National Laboratory and University of California, Berkeley, California 94720, USA

${ }^{48}$ California State University, Fresno, California 93740, USA

${ }^{49}$ University of California, Riverside, California 92521, USA

${ }^{50}$ Florida State University, Tallahassee, Florida 32306, USA

${ }^{51}$ Fermi National Accelerator Laboratory, Batavia, Illinois 60510, USA

${ }^{52}$ University of Illinois at Chicago, Chicago, Illinois 60607, USA

${ }^{53}$ Northern Illinois University, DeKalb, Illinois 60115, USA

${ }^{54}$ Northwestern University, Evanston, Illinois 60208, USA

${ }^{55}$ Indiana University, Bloomington, Indiana 47405, USA

${ }^{56}$ University of Notre Dame, Notre Dame, Indiana 46556, USA

${ }^{57}$ Purdue University Calumet, Hammond, Indiana 46323, USA

${ }^{58}$ Iowa State University, Ames, Iowa 50011, USA

${ }^{59}$ University of Kansas, Lawrence, Kansas 66045, USA

${ }^{60}$ Kansas State University, Manhattan, Kansas 66506, USA

${ }^{61}$ Louisiana Tech University, Ruston, Louisiana 71272, USA

${ }^{62}$ University of Maryland, College Park, Maryland 20742, USA

${ }^{63}$ Boston University, Boston, Massachusetts 02215, USA

${ }^{64}$ Northeastern University, Boston, Massachusetts 02115, USA

${ }^{65}$ University of Michigan, Ann Arbor, Michigan 48109, USA

${ }^{66}$ Michigan State University, East Lansing, Michigan 48824, USA

${ }^{67}$ University of Mississippi, University, Mississippi 38677, USA

${ }^{68}$ University of Nebraska, Lincoln, Nebraska 68588, USA

${ }^{69}$ Princeton University, Princeton, New Jersey 08544, USA

${ }^{70}$ State University of New York, Buffalo, New York 14260, USA

${ }^{71}$ Columbia University, New York, New York 10027, USA

${ }^{72}$ University of Rochester, Rochester, New York 14627, USA

${ }^{73}$ State University of New York, Stony Brook, New York 11794, USA

${ }^{74}$ Brookhaven National Laboratory, Upton, New York 11973, USA

${ }^{75}$ Langston University, Langston, Oklahoma 73050, USA

${ }^{76}$ University of Oklahoma, Norman, Oklahoma 73019, USA

${ }^{77}$ Oklahoma State University, Stillwater, Oklahoma 74078, USA

${ }^{78}$ Brown University, Providence, Rhode Island 02912, USA

${ }^{79}$ University of Texas, Arlington, Texas 76019, USA

${ }^{80}$ Southern Methodist University, Dallas, Texas 75275, USA

${ }^{81}$ Rice University, Houston, Texas 77005, USA

${ }^{82}$ University of Virginia, Charlottesville, Virginia 22901, USA

${ }^{83}$ University of Washington, Seattle, Washington 98195, USA

(Received 31 October 2007; revised manuscript received 11 December 2007; published 14 February 2008)

We present the first model-independent measurement of the helicity of $W$ bosons produced in top quark decays, based on a $1 \mathrm{fb}^{-1}$ sample of candidate $t \bar{t}$ events in the dilepton and lepton plus jets channels collected by the D0 detector at the Fermilab Tevatron $p \bar{p}$ Collider. We reconstruct the angle $\theta^{*}$ between the momenta of the down-type fermion and the top quark in the $W$ boson rest frame for each top quark decay. A fit of the resulting $\cos \theta^{*}$ distribution finds that the fraction of longitudinal $W$ bosons 
$f_{0}=0.425 \pm 0.166$ (stat) \pm 0.102 (syst) and the fraction of right-handed $W$ bosons $f_{+}=0.119 \pm$

0.090 (stat) \pm 0.053 (syst), which is consistent at the $30 \%$ C.L. with the standard model.

The top quark is by far the heaviest of the known fermions and is the only one that has a Yukawa coupling to the Higgs boson of order unity in the standard model (SM). In the SM, the top quark decays via the $V-A$ charged-current interaction, almost always to a $W$ boson and a $b$ quark. We search for evidence of new physics in the $t \rightarrow W b$ decay by measuring the helicity of the $W$ boson. A different Lorentz structure of the $t \rightarrow W b$ interaction would alter the fractions of $W$ bosons produced in each polarization state from the SM values of $0.697 \pm 0.012$ [1] and $3.6 \times 10^{-4}$ [2] for the longitudinal fraction $f_{0}$ and right-handed fraction $f_{+}$, respectively, at the world average top quark mass $m_{t}$ of $172.5 \pm 2.3 \mathrm{GeV}$ [3].

In this Letter, we report a simultaneous measurement of $f_{0}$ and $f_{+}$(the negative helicity fraction $f_{-}$is then fixed by the requirement that $\left.f_{-}+f_{0}+f_{+}=1\right)$. This is the first model-independent $W$ boson helicity measurement. A measurement of the $W$ boson helicity fractions that differs significantly from the SM values would be an unambiguous indication of new physics [4-6]. In addition, the model-independent $W$ boson helicity measurement can be combined with measurements of single top production cross sections to fully specify the $t b W$ vertex [7].

Measurements of the $b \rightarrow s \gamma$ decay rate assuming the absence of gluonic penguin contributions have indirectly limited the $V+A$ contribution in top quark decays to less than a few percent [8]. Direct measurements of the longitudinal fraction $\left(f_{+}\right.$set to zero) found $f_{0}=0.85_{-0.23}^{+0.16}$ [9] and $f_{0}=0.56 \pm 0.31$ [10]. Direct measurements of $f_{+}\left(f_{0}\right.$ set to 0.7 ) have found $f_{+}=-0.02 \pm 0.08$ [11] and $f_{+}=$ $0.06 \pm 0.10$ [12]. The analysis presented here improves upon that reported in Ref. [12] by using a larger data set, employing enhanced event selection techniques, making use of hadronic $W$ boson decays, and introducing the model-independent analysis in which $f_{0}$ and $f_{+}$are varied independently.

The angular distribution of the down-type decay products of the $W$ boson (charged lepton or $d, s$ quark) can be described by introducing the angle $\theta^{*}$ between the $W$ boson's momentum in the top quark rest frame and the down-type fermion's momentum in the $W$ boson rest frame. The dependence of the distribution of $\cos \theta^{*}$ on the $W$ boson helicity fractions,

$$
\omega(c) \propto 2\left(1-c^{2}\right) f_{0}+(1-c)^{2} f_{-}+(1+c)^{2} f_{+},
$$

where $c=\cos \theta^{*}$, forms the basis for our measurement. We proceed by selecting a data sample enriched in $t \bar{t}$ events, reconstructing the four vectors of the two top quarks and their decay products, and then calculating $\cos \theta^{*}$. The down-type fermions in leptonic $W$ boson decays are the charged leptons. For hadronic $W$ boson decays, we choose a $W$ boson daughter jet at random to calculate $\cos \theta^{*}$. Since this introduces a sign ambiguity into the calculation, we consider only $\left|\cos \theta^{*}\right|$ for hadronic $W$ boson decays. The $\left|\cos \theta^{*}\right|$ variable does not discriminate between left- and right-handed $W$ bosons, but adds information for determining the fraction of longitudinal $W$ bosons. These distributions in $\cos \theta^{*}$ are compared with templates for different $W$ boson helicity models, accounting for background and reconstruction effects, using a binned maximum likelihood method.

This measurement uses a data sample recorded with the D0 experiment [13] that corresponds to an integrated luminosity of about $1 \mathrm{fb}^{-1}$ of $p \bar{p}$ collisions at $\sqrt{s}=$ $1.96 \mathrm{TeV}$. The data sample consists of $t \bar{t}$ candidate events from the lepton plus jets $(\ell+$ jets) decay channel $t \bar{t} \rightarrow$ $W^{+} W^{-} b \bar{b} \rightarrow \ell \nu q q^{\prime} b \bar{b}$ and the dilepton channel $t \bar{t} \rightarrow$ $W^{+} W^{-} b \bar{b} \rightarrow \ell \nu \ell^{\prime} \nu^{\prime} b \bar{b}$, where $\ell$ and $\ell^{\prime}$ are electrons or muons. The $\ell+$ jets final state is characterized by one charged lepton, at least four jets, and large missing transverse energy $\left(\mathscr{E}_{T}\right)$. The dilepton final state is characterized by two charged leptons, at least two jets, and large $\mathbb{E}_{T}$. In both final states, at least two of the jets are $b$ jets.

The trigger requirements vary depending on the $t \bar{t}$ decay channel: for $\ell+$ jets events, both a jet and a lepton are required at trigger level, for $e e$ events a lepton is required, and for $e \mu$ and $\mu \mu$ events either a lepton or jet is required. The $\ell+$ jets event selection [14] requires an isolated charged lepton with transverse momentum $p_{T}>20 \mathrm{GeV}$, no other charged lepton with $p_{T}>15 \mathrm{GeV}$ in the event, $\mathscr{E}_{T}>20 \mathrm{GeV}$, and at least four jets. In the dilepton channel, events are required to have two charged leptons with opposite charge and $p_{T}>15 \mathrm{GeV}$ and two or more jets. Electrons are required to have pseudorapidity [15] $|\eta|<$ 1.1 in the $\ell+$ jets channel and $|\eta|<1.1$ or $1.5<|\eta|<$ 2.5 in the dilepton channel, and are identified by their energy deposition, isolation, and shower shape in the calorimeter, and information from the tracking system [14]. Muons are identified using information from the muon and tracking systems and must have little surrounding calorimeter energy or charged track momentum. They are required to have $|\eta|<2$.0. Jets are reconstructed using a cone algorithm with cone radius 0.5 [16] and are required to have rapidity $|y|<2.5$ and $p_{T}>20 \mathrm{GeV}$. The $\not_{T}$ is calculated from the vector sum of calorimeter cell energies, corrected to account for the response of the calorimeter to jets and electrons and also for the momenta of identified muons.

We simulate $t \bar{t}$ signal events with $m_{t}=172.5 \mathrm{GeV}$ for different values of $f_{+}$with the ALPGEN Monte Carlo (MC) 
program [17] for the parton-level process (leading order) and PYTHIA [18] for gluon radiation and subsequent hadronization. We generate samples corresponding to each of the three $W$ boson helicity configurations by reweighting the generated $\cos \theta^{*}$ distributions.

Backgrounds in the $\ell+$ jets channel arise mainly from $W+$ jets production and multijet production. In the dilepton channel, backgrounds arise from processes such as $W W+$ jets or $Z+$ jets. The MC samples used to model background events with real leptons are also generated using ALPGEN and PYTHIA. Both the signal and background MC samples are passed through a GEANT3 [19] simulation of the detector response and reconstructed with the same algorithms used for data. In the $\ell+$ jets channel we estimate the number $N_{\mathrm{mj}}$ of multijet background events from data, using the technique described in Ref. [14]. We calculate $N_{\mathrm{mj}}$ for each bin in the $\cos \theta^{*}$ distribution from the data sample to obtain the multijet $\cos \theta^{*}$ templates.

To increase the signal purity, a multivariate likelihood discriminant $\mathcal{D}$ [14] with values in the range 0 to 1 is calculated using input variables which exploit differences in kinematics and jet flavor. The kinematic variables make use of the fact that jets and leptons in signal events tend to have larger $p_{T}$, be more central, and be distributed more spherically in $\eta-\phi$ space than those in background. Signal events also have at least two $b$ jets, while background events usually have none. The likelihood of a given jet arising from a $b$ quark is estimated using the output value $N N_{b}$ of a neural network that combines several features of the tracks within the jet [20].

The discriminant is built separately for each of the five final states considered, using the method described in Refs. [14,21]. An example of the distributions of signal, background and data events in $\mathcal{D}$ is shown in Fig. 1.

We perform a binned Poisson maximum likelihood fit to compare the observed distribution of events in $\mathcal{D}$ to the sum of the distributions expected from $t \bar{t}$ and background

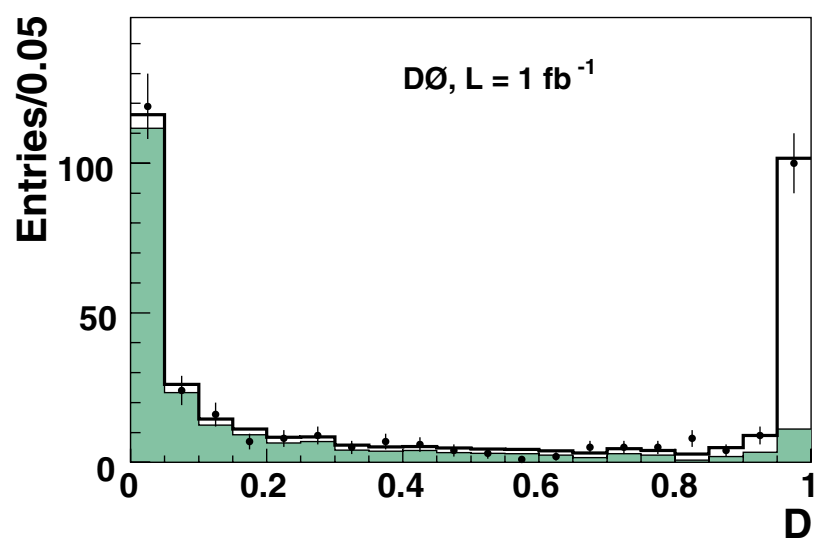

FIG. 1 (color online). Distribution of $\mathcal{D}$ for data (points with error bars), background (shaded histogram), and signal plus background (open histogram) in the $e+$ jets channel. events. In the $\ell+$ jets channels, $N_{\mathrm{mj}}$ is constrained to the expected value within the known uncertainty, while in the dilepton channels the ratio of the various background sources is fixed to the expectation from the cross sections times efficiency of the kinematic selection. The likelihood is then maximized with respect to the numbers of $t \bar{t}$ and background events, which are multiplied by the efficiency for the $\mathcal{D}$ selection to determine the composition of the sample used for measuring the $W$ boson helicity fractions. Table I lists the composition of each sample as well as the number of observed events in the data.

The top quark and $W$ boson four-momenta in the selected $\ell+$ jets events are reconstructed using a kinematic fit which is subject to the following constraints: two jets must form the invariant mass of the $W$ boson [22], the charged lepton and the $\mathscr{E}_{T}$ together with the neutrino $p_{z}$ component must form the invariant mass of the $W$ boson, and the masses of the two reconstructed top quarks must be $172.5 \mathrm{GeV}$. The four highest- $p_{T}$ jets in each event are used in the fit, and among the 12 possible jet combinations, the solution with the maximal probability, considering both the $\chi^{2}$ from the kinematic fit and the $N N_{b}$ values of the four jets, is chosen. The $\cos \theta^{*}$ distributions for leptonic and hadronic $W$ boson decays obtained in the $\ell+$ jets data after the full selection are shown in Figs. 2(a) and 2(b).

Since the two neutrinos in the dilepton final state are not detected, the system is kinematically underconstrained. However, if $m_{t}$ is assumed, the kinematics can be solved algebraically with a fourfold ambiguity in addition to the twofold ambiguity in pairing jets with charged leptons. For each of the two leading jets, we calculate the value of $\cos \theta^{*}$ resulting from each solution with each of the two charged leptons associated with the jet. To explore the phase space consistent with the measured jet and charged lepton energies, we fluctuate them according to their resolution many times, and repeat the above procedure for each fluctuation. The average of the values arising from each solution ambiguity and event fluctuation is taken as $\cos \theta^{*}$ for that jet. The $\cos \theta^{*}$ distribution obtained in dilepton data is shown in Fig. 2(c).

To extract $f_{0}$ and $f_{+}$, we compute the binned Poisson likelihood $L\left(f_{0}, f_{+}\right)$for the data to be consistent with the sum of templates for negative-, zero-, and positive-helicity signal and background at any given value for these fractions. The background normalization is constrained to be consistent within uncertainties with the expected value by a

TABLE I. Summary of the multivariate selection and number of selected events for the $t \bar{t}$ final states used in this analysis. The uncertainties are statistical only.

\begin{tabular}{lccc}
\hline \hline & $\ell+$ jets & $e \mu$ & $e e$ and $\mu \mu$ \\
\hline$t \bar{t}$ Purity before $\mathcal{D}$ selection $(\%)$ & $41 \pm 3$ & $66 \pm 13$ & $2.0 \pm 0.4$ \\
Background after $\mathcal{D}$ selection & $54.1 \pm 6.9$ & $9.9 \pm 2.5$ & $7.0 \pm 0.1$ \\
Data events after $\mathcal{D}$ selection & 288 & 45 & 30 \\
\hline \hline
\end{tabular}



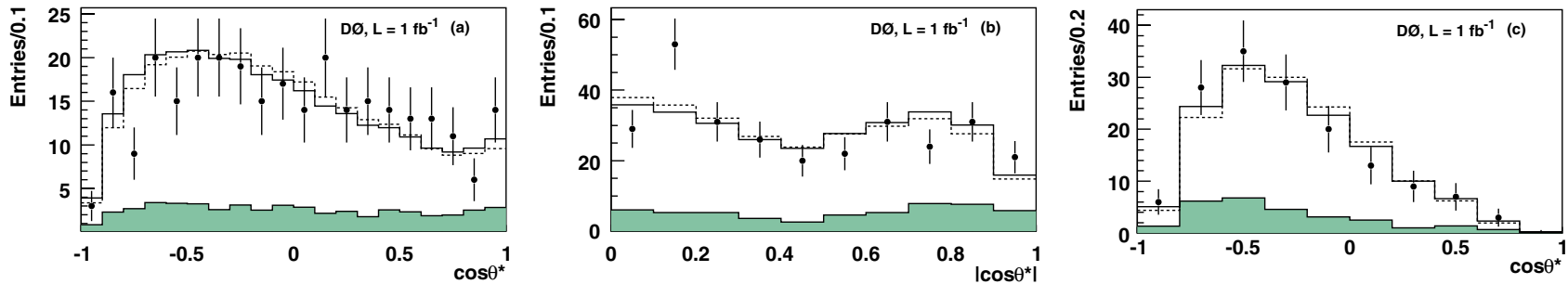

FIG. 2 (color online). Comparison of the $\cos \theta^{*}$ distribution in data (points with error bars) and the global best-fit model (solid open histograms) for (a) leptonic $W$ boson decays in $\ell+$ jets events, (b) hadronic $W$ boson decays in $\ell+$ jets events, and (c) dilepton events. The dashed open histograms show the SM expectation, and the shaded histograms represent the background contribution. Each $\ell+$ jets event provides an entry to both (a) and (b), while each dilepton event provides two entries to (c).

Gaussian term in the likelihood. The fit also accounts for the differences in selection efficiency for $t \bar{t}$ events with different $W$ helicity configurations [23].

Systematic uncertainties are evaluated in ensemble tests by varying the parameters that can affect the measurement. Ensembles are formed by drawing events from a model with the parameter under study varied. These are compared to the standard $\cos \theta^{*}$ templates in a maximum likelihood fit. The average shifts in the resulting $f_{0}$ and $f_{+}$values are taken as the systematic uncertainty and are shown in Table II. The total systematic uncertainty is then taken into account in the likelihood by convoluting the likelihood with a Gaussian with a width that corresponds to the total systematic uncertainty. The mass of the top quark is varied by $\pm 2.3 \mathrm{GeV}$, and the jet reconstruction efficiency, energy calibration, and $b$ fragmentation parameters by $\pm 1 \sigma$ around their nominal values. The $t \bar{t}$ model uncertainty is studied by comparing $t \bar{t}$ events generated by PYTHIA to the standard ALPGEN samples, considering samples with a different model for the underlying event and ones in which only a single primary vertex is reconstructed. Effects of mismodeling the background distribution in $\cos \theta^{*}$ are assessed by comparing data to the background model for events with low $\mathcal{D}$ values. The uncertainty due to template statistics is evaluated by fluctuating the templates according to their statistical uncertainties and repeating the fit to the data for each fluctuation. Uncertainties due to jet resolution, jet flavor composition in the background, the

TABLE II. Summary of the major systematic uncertainties on $f_{0}$ and $f_{+}$in the model-independent fit.

\begin{tabular}{lcc}
\hline \hline \multicolumn{1}{c}{ Source } & Uncertainty $\left(f_{0}\right)$ & Uncertainty $\left(f_{+}\right)$ \\
\hline Top mass & 0.009 & 0.018 \\
Jet reconstruction eff. & 0.021 & 0.010 \\
Jet energy calibration & 0.012 & 0.019 \\
$b$ fragmentation & 0.016 & 0.010 \\
$t \bar{t}$ model & 0.068 & 0.032 \\
Background model & 0.049 & 0.016 \\
Template statistics & 0.049 & 0.025 \\
Total & 0.102 & 0.053 \\
\hline \hline
\end{tabular}

modeling of the $N N_{b}$ variable, and parton distribution functions are all found to be less than 0.01 for both $f_{0}$ and $f_{+}$.

The measured values of $f_{0}$ and $f_{+}$are

$$
\begin{aligned}
& f_{0}=0.425 \pm 0.166(\text { stat }) \pm 0.102(\text { syst }) \\
& f_{+}=0.119 \pm 0.090(\text { stat }) \pm 0.053(\text { syst })
\end{aligned}
$$

with a correlation coefficient of -0.83 . The inclusion of the $\left|\cos \theta^{*}\right|$ measurement from hadronic $W$ boson decays improves the uncertainties on $f_{0}$ and $f_{+}$by about $20 \%$ relative to those obtained using only the leptonic decays. The $68 \%$ and $95 \%$ C.L. contours from the fit, including systematic uncertainties, are shown in Fig. 3. The data indicate fewer longitudinal and more right-handed $W$ bosons than the SM predicts, but the difference is not significant as there is a 30\% chance of observing a larger discrepancy given the statistical and systematic uncertainties in the measurement.

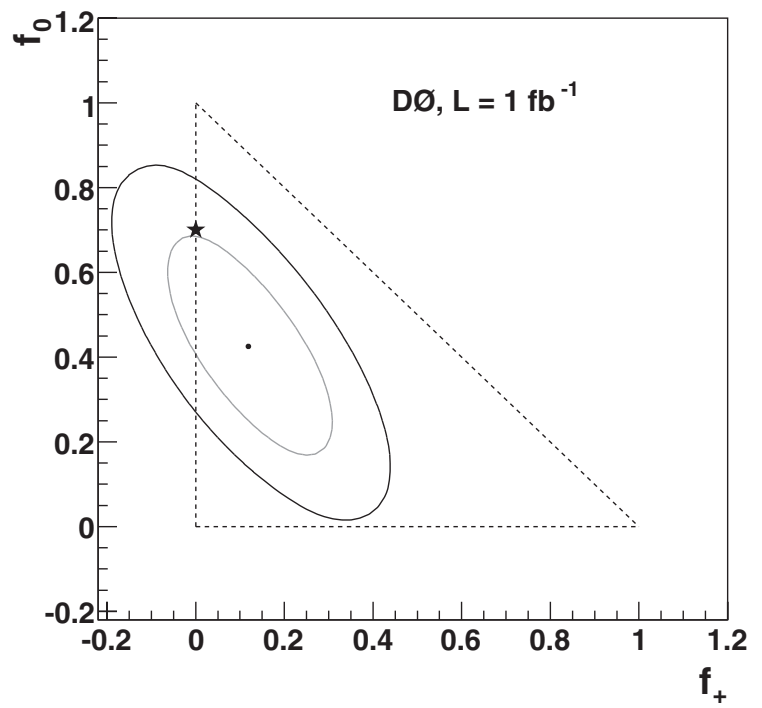

FIG. 3. Result of the model-independent $W$ boson helicity fit. The ellipses are the $68 \%$ and $95 \%$ C.L. contours, the triangle borders the physically allowed region where $f_{0}$ and $f_{+}$sum to one or less, and the star denotes the SM values. 
If we fix $f_{+}$to the SM value, we find

$$
f_{0}=0.619 \pm 0.090(\text { stat }) \pm 0.052 \text { (syst), }
$$

and if $f_{0}$ is fixed to the SM value we find

$$
\begin{aligned}
& f_{+}=-0.002 \pm 0.047 \text { (stat) } \pm 0.047 \text { (syst) } \\
& \left(f_{+}<0.13 \text { at } 95 \% \text { C.L. }\right) .
\end{aligned}
$$

Equations (3) and (4) are directly comparable to previous measurements [9-12].

In summary, we have measured the helicity fractions of $W$ bosons in $t \bar{t}$ decays in the $\ell+$ jets and dilepton channels with a model-independent fit and find $f_{0}=0.425 \pm$ 0.166 (stat) \pm 0.102 (syst) and $f_{+}=0.119 \pm 0.090$ (stat) \pm 0.053 (syst). This is the first such measurement reported and is consistent at the $30 \%$ level with the SM values of $f_{0}=0.697 \pm 0.012$ [1] and $f_{+}=3.6 \times 10^{-4}$ [1]. We have also measured $f_{0}$ and $f_{+}$in a model-dependent fit and find that they are consistent with the SM values.

We thank the staffs at Fermilab and collaborating institutions, and acknowledge support from the DOE and NSF (USA); CEA and No. CNRS/IN2P3 (France); FASI, Rosatom and RFBR (Russia); CAPES, CNPq, FAPERJ, FAPESP and FUNDUNESP (Brazil); DAE and DST (India); Colciencias (Colombia); CONACyT (Mexico); KRF and KOSEF (Korea); CONICET and UBACyT (Argentina); FOM (The Netherlands); Science and Technology Facilities Council (United Kingdom); MSMT and GACR (Czech Republic); CRC Program, CFI, NSERC and WestGrid Project (Canada); BMBF and DFG (Germany); SFI (Ireland); The Swedish Research Council (Sweden); CAS and CNSF (China); and the Alexander von Humboldt Foundation.

*Visitor from Augustana College, Sioux Falls, SD, USA.

'Visitor from The University of Liverpool, Liverpool, United Kingdom.

${ }^{\ddagger}$ Visitor from ICN-UNAM, Mexico City, Mexico.

${ }^{\S}$ Visitor from II. Physikalisches Institut, Georg-AugustUniversity, Göttingen, Germany.

${ }^{\| V}$ Visitor from Helsinki Institute of Physics, Helsinki, Finland.
"Deceased.

[1] G. L. Kane, G. A. Ladinsky, and C.-P. Yuan, Phys. Rev. D 45, 124 (1992); R. H. Dalitz and G. R. Goldstein, Phys. Rev. D 45, 1531 (1992); C. A. Nelson et al., Phys. Rev. D 56, 5928 (1997).

[2] M. Fischer et al., Phys. Rev. D 63, 031501(R) (2001).

[3] Tevatron Electroweak Working Group, arXiv:hep-ex/ 0603039.

[4] J. Cao et al., Phys. Rev. D 68, 054019 (2003).

[5] Y. M. Nie et al., Phys. Rev. D 71, 074018 (2005).

[6] X. Wang, Q. Zhang, and Q. Qiao, Phys. Rev. D 71, 014035 (2005).

[7] C.-R. Chen, F. Larios, and C.-P. Yuan, Phys. Lett. B 631, 126 (2005); V. M. Abazov et al. (D0 Collaboration), Phys. Rev. Lett. 98, 181802 (2007).

[8] K. Fujikawa and A. Yamada, Phys. Rev. D 49, 5890 (1994); P. Cho and M. Misiak, Phys. Rev. D 49, 5894 (1994); C. Jessop, Report No. SLAC-PUB-9610, 2002.

[9] A. Abulencia et al. (CDF Collaboration), Phys. Rev. D 75, 052001 (2007).

[10] V. M. Abazov et al. (D0 Collaboration), Phys. Lett. B 617, 1 (2005).

[11] A. Abulencia et al. (CDF Collaboration), Phys. Rev. Lett. 98, 072001 (2007).

[12] V. M. Abazov et al. (D0 Collaboration), Phys. Rev. D 75, 031102(R) (2007).

[13] V.M. Abazov et al. (D0 Collaboration), Nucl. Instrum. Methods Phys. Res., Sect. A 565, 463 (2006).

[14] V. M. Abazov et al. (D0 Collaboration), Phys. Lett. B 626, 45 (2005).

[15] Rapidity $y$ and pseudorapidity $\eta$ are defined as functions of the polar angle $\theta$ with respect to the proton beam and the parameter $\beta$ as $y(\theta, \beta) \equiv \frac{1}{2} \ln [(1+\beta \cos \theta) /(1-$ $\beta \cos \theta)]$ and $\eta(\theta) \equiv y(\theta, 1)$, where $\beta$ is the ratio of a particle's momentum to its energy.

[16] G. C. Blazey et al., arXiv:hep-ex/0005012.

[17] M. L. Mangano et al., J. High Energy Phys. 07 (2003) 001.

[18] T. Sjöstrand et al., Comput. Phys. Commun. 135, 238 (2001).

[19] R. Brun and F. Carminati, CERN Program Library Long Writeup W5013, 1994 (unpublished).

[20] T. Scanlon, Ph.D. thesis, University of London (report No. FERMILAB-THESIS-2006-43, 2006).

[21] B. Abbott et al. (D0 Collaboration), Phys. Rev. D 58, 052001 (1998).

[22] W. M. Yao et al. (Particle Data Group), J. Phys. G 33, 1 (2006).

[23] D. Glenzinski and B. Kilminster (private communication). 